\title{
Thromboelastography (TEG) and thromboelastometry (ROTEM) for trauma induced coagulopathy in adult trauma patients with bleeding (Protocol)
}

Hunt H, Hyde C, Stanworth S, Curry N, Perel P, Woolley T, Cooper C, Ukoumunne O

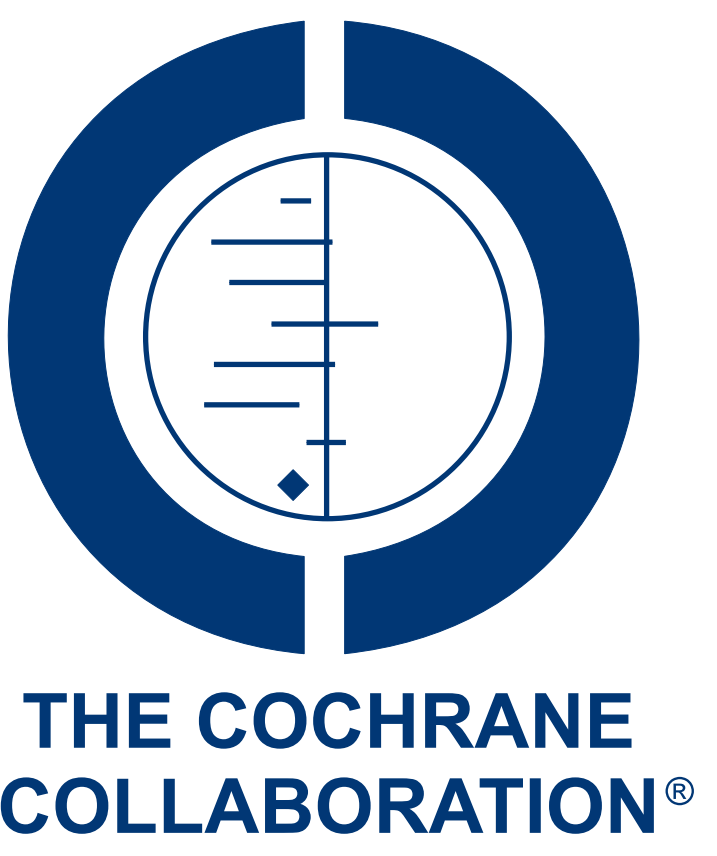

This is a reprint of a Cochrane protocol, prepared and maintained by The Cochrane Collaboration and published in The Cochrane Library 2013, Issue 3

Thromboelastography (TEG) and thromboelastometry (ROTEM) for trauma

- induced coagulopathy in adult trauma patients with bleeding (Protocol)

Copyright @ 2013 The Cochrane Collaboration. Published by John Wiley \& Sons, Ltd. 
0induced coagulopathy in adult trauma patients with bleeding

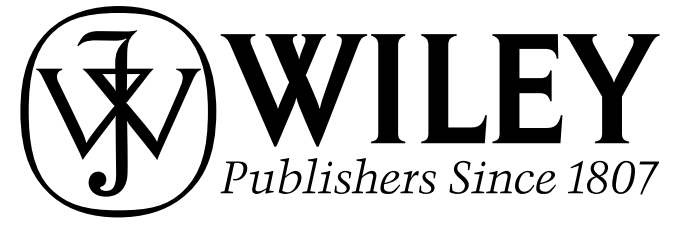

Thromboelastography (TEG) and thromboelastometry (ROTEM) for trauma

induced coagulopathy in adult trauma patients with bleeding (Protocol)

Copyright @ 2013 The Cochrane Collaboration. Published by John Wiley \& Sons, Ltd. 
TABLE OF CONTENTS

HEADER . . . . . . . . . . . . . . . . . . . . . . . . . . . . . . . . . 1

ABSTRACT . . . . . . . . . . . . . . . . . . . . . . . . . . . . . . . . . . . . . . 1

BACKGROUND . . . . . . . . . . . . . . . . . . . . . . . . . . . . . . . . . . . . . . . . . . .

Figure 1. . . . . . . . . . . . . . . . . . . . . . . . . . . . . . . . . . . . . . 2

OBJECTIVES . . . . . . . . . . . . . . . . . . . . . . . . . . . . . . . . . . . . . . . . . . . . .

METHODS . . . . . . . . . . . . . . . . . . . . . . . . . . . . . . . . . . . . . . 3

Figure 2. . . . . . . . . . . . . . . . . . . . . . . . . . . . . . . . . . . . . . 4

ACKNOWLEDGEMENTS . . . . . . . . . . . . . . . . . . . . . . . . . . . . . . . . 6

REFERENCES . . . . . . . . . . . . . . . . . . . . . . . . . . . . . . . . . . . . . . 7

ADDITIONAL TABLES . . . . . . . . . . . . . . . . . . . . . . . . . . . . . . . . . . . 8

APPENDICES . . . . . . . . . . . . . . . . . . . . . . . . . . . . . . . . . . . . . . 8

Figure 3. . . . . . . . . . . . . . . . . . . . . . . . . . . . . . . . . . . . 10

Figure 4. . . . . . . . . . . . . . . . . . . . . . . . . . . . . . . . . . . . . . 14

Figure 5. . . . . . . . . . . . . . . . . . . . . . . . . . . . . . . . . . . . . .

Figure 6. . . . . . . . . . . . . . . . . . . . . . . . . . . . . . . . . . . . . . .

CONTRIBUTIONS OF AUTHORS . . . . . . . . . . . . . . . . . . . . . . . . . . . . . . . . . . . . . . . . .

DECLARATIONS OF INTEREST . . . . . . . . . . . . . . . . . . . . . . . . . . . . . . . . 17

SOURCES OF SUPPORT . . . . . . . . . . . . . . . . . . . . . . . . . . . . . . . . . . 17

Thromboelastography (TEG) and thromboelastometry (ROTEM) for trauma 


\title{
Thromboelastography (TEG) and thromboelastometry (ROTEM) for trauma induced coagulopathy in adult trauma patients with bleeding
}

\author{
Harriet Hunt ${ }^{1}$, Chris Hyde ${ }^{1}$, Simon Stanworth ${ }^{2}$, Nicola Curry ${ }^{3}$, Pablo Perel ${ }^{4}$, Tom Woolley ${ }^{5}$, Chris Cooper ${ }^{1}$, Obioha Ukoumunne ${ }^{6}$ \\ ${ }^{1}$ Peninsula Technology Assessment Group (PenTAG), University of Exeter Medical School, University of Exeter, Exeter, UK. \\ ${ }^{2}$ Haematology/Transfusion Medicine, NHS Blood and Transplant, Oxford, UK. ${ }^{3}$ NHS Blood and Transplant, John Radcliffe Hospital, \\ Oxford, UK. ${ }^{4}$ Cochrane Injuries Group, London School of Hygiene \& Tropical Medicine, London, UK. ${ }^{5}$ Department of Military \\ Anaesthesia and Critical Care, Royal Centre for Defence Medicine ICT Centre, Birmingham, UK. ${ }^{6}$ PenCLAHRC, University of Exeter \\ Medical School, University of Exeter, Exeter, UK
}

Contact address: Harriet Hunt, Peninsula Technology Assessment Group (PenTAG), University of Exeter Medical School, University of Exeter, Exeter, UK. H.A.Hunt@exeter.ac.uk.

Editorial group: Cochrane Injuries Group.

Publication status and date: New, published in Issue 3, 2013.

Citation: Hunt H, Hyde C, Stanworth S, Curry N, Perel P, Woolley T, Cooper C, Ukoumunne O. Thromboelastography (TEG) and thromboelastometry (ROTEM) for trauma- induced coagulopathy in adult trauma patients with bleeding. Cochrane Database of Systematic Reviews 2013, Issue 3. Art. No.: CD010438. DOI: 10.1002/14651858.CD010438.

Copyright (C) 2013 The Cochrane Collaboration. Published by John Wiley \& Sons, Ltd.

\begin{abstract}
A B S T R A C T
This is the protocol for a review and there is no abstract. The objectives are as follows:

To determine the diagnostic accuracy of global tests of haemostatic function (thromboelastography (TEG) and thromboelastometry (ROTEM)) in adult trauma patients with bleeding.
\end{abstract}

Thromboelastography (TEG) and thromboelastometry (ROTEM) for trauma 


\section{B A C K G R O U N D}

\section{Target condition being diagnosed}

Trauma-induced coagulopathy (TIC) can be defined as an impairment of blood clotting occurring early after injury (Frith 2010). A diagnosis of TIC on admission carries a mortality rate amongst patients of up to $50 \%$, and is often associated with increased burdens of transfusion, greater risks of organ injury and septic complications, and longer stays in critical care (Brohi 2003; MacLeod 2003; Maegele 2007). Worldwide, trauma is the leading cause of mortality and disability in adults under the age of 36 years old (Hess 2009 ) and $40 \%$ of all trauma deaths (in the UK) are as a result of haemorrhage (Frith 2010), whilst shock and coagulopathy upon admission have both been independently associated with massive transfusion and increased mortality (Spinella 2009). Equally in the combat setting, bleeding is the largest cause of death on the battlefield (Holcomb 2007).

Various terms such as TIC, 'acute traumatic coagulopathy' (ATC) and 'acute coagulopathy of trauma shock' are used to describe these early coagulation changes. None of these terms have taken particular precedence and all are widespread within the trauma literature. For the purposes of this review we will use the term 'trauma-induced coagulopathy' (TIC) to describe the hypocoagulable changes that occur within the first 24 hours following injury due to a variety of different and highly interlinked causes, i.e. hypoperfusion, ongoing bleeding and consumption of clotting factors, haemodilution, acidosis, hypothermia and ATC. In the absence of embedded clinical consensus, the coagulopathic range we will be using is based on pro-thrombin time ratio (PTr) /International Normalized Ratio (INR). Anyone with a PTr/INR count of above 1.2, or above 1.5, is considered coagulopathic (further detail is given in the section on Reference standards). These figures were reached through review of the literature and discussion by the report authors, including experts in haematology and trauma medicine.
The aetiology of coagulopathy associated with trauma is not fully understood. In non-trauma situations, blood clots form through a chain of actions; first, platelets form a sticky clump (or 'white clot') on the blood vessel wall at the site of injury. This clot is weak, but soon a cascade of clotting proteins generates fibrin, a protein which meshes the platelets and some red blood cells together to produce a far stronger clot (or 'red clot'). This process is called coagulation, but it can become disordered - and this happens in around a quarter of trauma patients. The underlying reasons for this disruption are still unknown, but the combination of tissue damage and shock are contributory factors, as is the presence of hypoperfusion through severe blood loss (Barts \& The London 2011).

Early recognition of the nature of the clotting defect has been acknowledged as increasingly important to guide replacement of clotting factors alongside blood volume maintenance and red cell replacement. However, there are no validated methods to effectively guide therapy. This leads to both over-transfusion and under-transfusion, reduction in efficacy, increased wastage and exposure to risk. These issues can be exacerbated in disasters where timely availability of blood and component therapy is vital but severely resource constrained.

\section{Clinical pathway}

Standard blood tests - i.e. the 'current tests' - are performed as soon as possible on arrival to hospital (see Figure 1). There is no hierarchy of tests performed at admission, but rather a group of tests - i.e. activated partial thromboplastin time (APTT), PTr/INR and full blood count (FBC). The choice of these tests is very variable and follows local hospital practice. In some centres, especially across Europe, thromboelastography (TEG)/thromboelastometry (ROTEM) are standard tests. In the UK the use of TEG and ROTEM is increasing, but has - up until now - mainly been in the experimental and research fora.

Figure I. Clinical pathway for emergency department identification of trauma-induced coagulopathy.

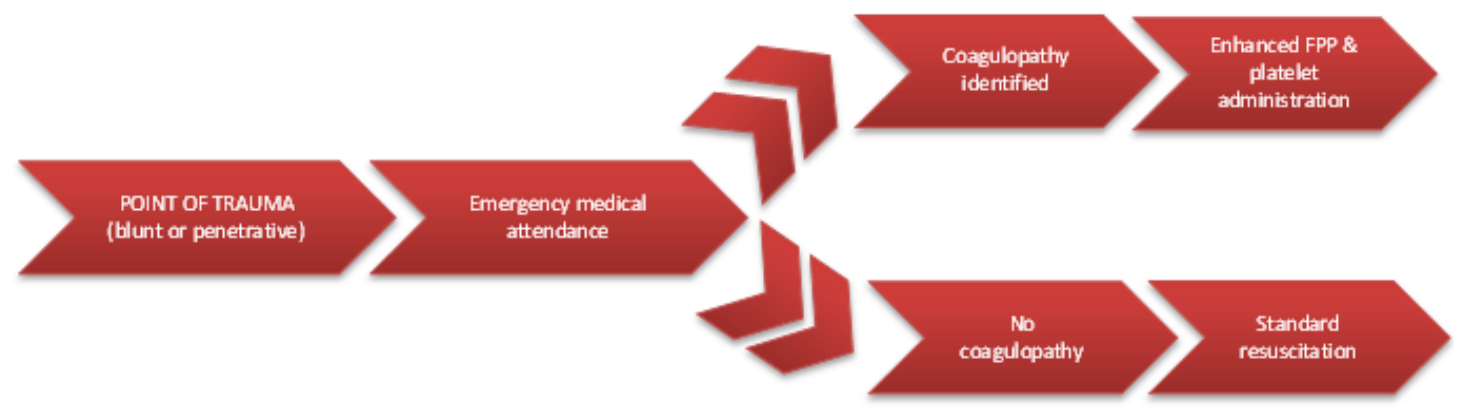

Thromboelastography (TEG) and thromboelastometry (ROTEM) for trauma

- induced coagulopathy in adult trauma patients with bleeding (Protocol)

Copyright (? 2013 The Cochrane Collaboration. Published by John Wiley \& Sons, Ltd. 


\section{Current tests}

Traditional measures of clotting such as platelet count, bleeding time, PT and APTT have some limitations in the context of managing trauma. Amongst these,

- platelet count provides numbers of how many platelets are present but gives no information about how they function;

- bleeding time measured through the application of a cuff

also assesses platelet function, but is impractical in the bleeding patient;

- fibrinogen readings allow measurement of fibrinogen levels in the blood, but this is specific to fibrinogen and does not give an overall indication of coagulopathic function;

- PT and APTT only provide a measure of time before initial thrombin generation, are performed on platelet-poor plasma, were designed to evaluate clotting factor deficiencies (not acquired coagulopathy), and are known to be poor predictors of bleeding in these circumstances (Dzik 2004).

In addition, evidence has suggested that APTT and PT are not able to provide an indication of when a patient is in a hypercoagulable state (Park 2009).

Despite these weaknesses, in practical terms PT remains the current standard of practice.

\section{Index tests}

Newer global haemostatic function technologies such as TEG and ROTEM enable 'point of care' measurement, using whole blood samples, of the initiation and progress of coagulation as well as final clot strength and lysis and the dynamics of clot formation. For the purposes of this study, TEG and ROTEM are being envisaged as a replacement test for traditional clotting tests. Both tests are currently used in routine clinical practice as both a diagnostic tool and to guide treatment.

Both TEG (trademark of Haemonetics Corporation, USA: www.haemonetics.com) and ROTEM (trademark of TEM International GmbH: www.rotem.de) work by measuring shear elastic modulus during clot formation and subsequent fibrinolysis. In both tests the whole blood sample is placed in a sample cup or 'cuvette' into which a cylindrical pin is immersed, leaving a small gap between the bottom of the pin and the base of the cuvette. The subsequent movement of the blood (designed to emulate sluggish circulation) is where the main difference lies between the two methods. When the sample blood begins to clot (i.e. fibrin begins to form, measured as clotting time or 'time to clot'), the movement of the pin becomes restricted with increasing firmness and this kinetic is transferred to the machinery of the TEG or ROTEM unit.

The next stage of the coagulation process is platelet aggregation, where platelets build in the blood vessel walls at the site of injury, and fibrin binds to the platelets which then forms a stronger clot, measured in both TEG and ROTEM in shear elasticity units as 'clot stability'. Eventually lysis - or clot break down - is measured, and a graphic is produced which represents haemostatic performance at all these stages: clotting time, clot formation, clot stability and lysis (see Appendix 1).

Whilst both TEG and ROTEM measure clotting time, clot formation, clot strengthening, amplitude of clot, maximum strength of clot, and clot lysis, they use slightly different terms or lettering to designate these features. We draw out these differences in detail in Appendix 2 and Table 1.

\section{Rationale}

This systematic review will form part of the evidence for a wider research programme which aims to improve outcomes for severely injured bleeding trauma patients, designed around the principle that early identification of patients who present with a traumainduced coagulopathy and effective, directed therapy will lead to improved outcomes, reduced complications, rationalised transfusions, reduced costs to the National Health Service (NHS) and a reduced logistical burden to the military and humanitarian organisations (such as the Red Cross) within austere combat environments. However, these tests require proper evaluation. Test accuracy studies have been conducted amongst evaluations thus far and should be systematically reviewed.

To complement this review we also propose to carry out a systematic review of prognosis studies linking measures from TEG/ ROTEM with patient outcome. This cannot be included in The Cochrane Library at present.

\section{O B J E C T I VES}

To determine the diagnostic accuracy of global tests of haemostatic function (thromboelastography (TEG) and thromboelastometry $($ ROTEM $)$ in adult trauma patients with bleeding.

\section{METHODS}

\section{Criteria for considering studies for this review}

\section{Types of studies}

We will include cross-sectional studies investigating the diagnostic test accuracy of TEG or ROTEM in patients with clinically suspected trauma-induced coagulopathy. We will expand the inclusion criteria to include case control studies if the number of sources retrieved is insufficient for a valid systematic review and possible meta-analysis. Pragmatically, we have set this level at less than 100 patients in total in the included studies. 


\section{Participants}

We will include adult trauma patients with clinically suspected trauma-induced coagulopathy. We will include studies in both military and civilian settings.

\section{Index tests}

Two global tests of haemostatic function will be used; TEG (thromboelastography - trademark of the Haemonetics Corporation, USA) and ROTEM (rotational thromboelastometry - trademark of TEM International $\mathrm{GmbH}$ ). Thresholds are indicated in Table 1.

\section{Target conditions}

The target condition will be trauma-induced coagulopathy defined by standard clotting times of pro-thrombin time ratio (PTr) and International Normalized Ratio (INR).

\section{Reference standards}

In the absence of embedded clinical consensus, the coagulopathic range we will be using is based on PTr/INR, with the lower limit of the range being a PTr/INR reading of 1.2 or greater (Frith 2010), and the upper limit of 1.5 or greater (Stainsby 2006). There is no upper limit to the range: anyone with a PTr/INR count of above 1.2 , or above 1.5 , is considered coagulopathic. These figures were reached through discussion by the report authors, including experts in haematology and trauma medicine. We will exclude studies using different cut-offs (i.e. not a PTr/INR reading of 1.2 or greater, or a PTr/INR reading of 1.5 or greater).

PTr differs from INR, although the final numbers may be the same. The PTr calculated varies depending on local thresholds and separate batches of different manufacturer's reagent involved in conducting the prothrombin time test. In an effort to standardise this measurement, the INR is calculated as the ratio of a patient's prothrombin time compared to a mean normal PTr (calculated by determining the mean of 30 or more patients who are representative of the local hospital population), computed to the power of the International Sensitivity Index (ISI), which is itself calculated by the manufacturer to give an indication of how each batch of tissue factor corresponds to an international reference. The equation for calculation is at Figure 2.

Figure 2. INR calculation.

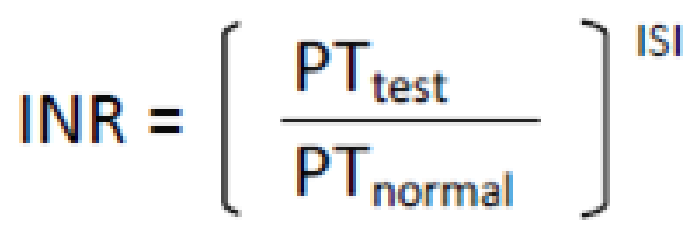

\section{Search methods for identification of studies}

We will use a sensitive search strategy to identify literature relating to the index tests for this review. This strategy will not be limited by language but will be limited by date to ' 1970 to current' and to 'human only' populations.

\section{Electronic searches}

We will search the following bibliographic resources:

- The Cochrane Library,

- British Nursing Index,

- Centre for Reviews and Dissemination databases,

- CINAHL,
- Conference Proceedings Citation Index - Science (CPCI-S),

- Conference Proceedings Citation Index - Social Science \& Humanities (CPCI-SSH),

- EMBASE,

- HMIC,

- MEDLINE in Process,

- MEDLINE,

- PsycINFO,

- Science Citation Index Expanded (SCI-EXPANDED),

- Social Sciences Citation Index (SSCI),

- LILACS,

- the Transfusion Evidence Library.

Thromboelastography (TEG) and thromboelastometry (ROTEM) for trauma

induced coagulopathy in adult trauma patients with bleeding (Protocol)

Copyright (? 2013 The Cochrane Collaboration. Published by John Wiley \& Sons, Ltd. 
We will search the following trial registers:

- Current Controlled Trials,

- Clinical Trials.Gov,

- the WHO International Trials Registry Platform via http:// www.who.int/ictrp/en/

We will search the following websites:

- Aggressive Research Intelligence Facility (ARIF) via http:// tinyurl.com/3u9tevp,

- C-EBLM,

- Cochrane Diagnostic Test Accuracy Working Group via http://srdta.cochrane.org/,

- Google,

- MEDION database via http://www.mediondatabase.nl,

- Haemonetics Corporation http://www.haemonetics.com/ en.aspx,

- TEM Innovations GmbH http://www.rotem.de/site/ index.php.

\section{Searching other resources}

We will conduct citation chasing on all studies included for full text screening. We will attempt to contact authors for any additional or supporting information.

For further details on the search, including the search strategy, please see Appendix 3.

\section{Data collection and analysis}

\section{Selection of studies}

CC will run the searches, collate the results and remove duplicates before transferring them to $\mathrm{HH}$ and $\mathrm{CH}$ for screening. All sources will be managed using Review Manager software version 5.2 (RevMan 2012). The inclusion criteria will be based on the Criteria for considering studies for this review. Decisions on inclusion/exclusion of studies will be made independently by two authors $(\mathrm{HH}$ and $\mathrm{CH})$ using piloted criteria. Disagreements will be resolved with reference to a third experienced author (SS and PP).

\section{Data extraction and management}

We will extract the following data (where available) into a bespoke data extraction table:

- Author, year of study, year of publication, journal reference;

- Study design and timing of data collection (prospective/ retrospective);

- Study population and participant characteristics (age, sex, setting - e.g. hospital, region, country, other details given)

- Trauma type:
- Blunt/penetrating,

- traumatic brain injury (TBI)/no TBI,

- Site of injury;

- Trauma severity as measured by:

○ Injury Severity Score (ISS),

- New ISS (NISS), and

- Trauma ISS (TRISS);

- Length of time from injury to admission;

- \% receiving massive transfusion (defined as $\geq 10$ units packed red blood cells (PRBC) in 24 hours, or the replacement of an equivalent amount of blood to an entire circulating blood volume of the patient within 24 hours (Doran 2010));

- Mean and interquartile range (IQR) number of units of blood and blood components (fresh frozen plasma (FFP), platelets and cryoprecipitate) transfused;

- Temperature (\% hypothermic at 33 degrees or below), systolic blood pressure (\% shocked), and base deficit (\% with hypoperfusion) on admission;

- Duration of bleed at point of testing;

- Reference test used (PTr/INR) and any other measures taken (of, for example, PT, APTT, fibrinogen level, platelet count, fibrinogen degradation products);

- Index test used (TEG/ROTEM) and version of device;

- Any details about device reliability;

- When tests were carried out in treatment phase (i.e. pre/ post transfusion, timings);

- Data from the 2x2 table will be extracted where presented, i.e. true positives, false positives, true negatives and false negatives;

- QUADAS-2 items (see Appendix 3).

Where available, we will record variability between operators and assay conditions. Particular care is likely to be required on many of these items (index test and reference standard) because of lack of standardisation. Two authors $(\mathrm{HH}$ and $\mathrm{CH}$ ) will pilot the extraction form using two primary diagnostic studies. A third author (NC) will resolve disagreements. The form will be accompanied by a briefing document explaining how it should be used. Data will be extracted by one author $(\mathrm{HH})$ and checked by a second $(\mathrm{CH})$, with a third author (NC) providing moderation as required.

\section{Assessment of methodological quality}

We will carry out quality assessment using a checklist approach to assess the quality of primary studies based on the QUADAS-2 instrument (see Appendix 2) in line with advice given in Reitsma 2009. We will independently score each item as 'Yes', 'No' or 'Unclear' as recommended by the Cochrane Handbook for Diagnostic Test Accuracy Reviews (http://srdta.cochrane.org/). A categorisation of 'unclear' will generally be considered a marker of poor quality, so care will be taken to account for the possibility that failing to report an item was reasonable given the circumstances in which the study was conducted. We will present results narratively 
in the text, and in an appropriate graphic representation of quality assessment (such as a table).

\section{Statistical analysis and data synthesis}

We will consider the accuracy of TEG and ROTEM compared to the reference standard as detailed above, considering $>1.2$ and $>$ 1.5 separately. We will not formally compare TEG and ROTEM although we will explore test type as a potential explanation for any heterogeneity. Results will be the components of the $2 \times 2$ table, sensitivity and specificity and their $95 \%$ confidence interval $(\mathrm{CI})$. These will be tabulated and presented graphically (forest plots and receiver operating characteristic (ROC) space). Our initial approach to analysis is likely to be qualitative, with conclusions based on patterns of results. Quantitative meta-analysis may be appropriate where the quantity and nature of the included studies permit. If meta-analysis is possible, our approach will be to calculate a summary ROC (sROC) curve using an hierarchical summary ROC (HSROC) model. We will also consider using a bivariate model depending on the data, but a priori uncertainty about thresholds, and the likelihood of implicit thresholds, suggest the HSROC model may be slightly preferable in the first instance. We will generate a summary of results table. If feasible and appropriate, translation of any summary results into natural frequencies and other metrics such as predictive values will be considered to facilitate improved understanding to readers.

We will tabulate and comment on the number of uninterpretable results. We will carry out analysis and presentation of results in line with advice in Chapter 10 of the Cochrane Handbook for Systematic Reviews of Diagnostic Test Accuracy.

We will scrutinise carefully all the included studies for any further investigation of discrepant results between the index test and reference standards (False Positives (FP) and False Negatives (FN)), ideally based on independent clinical review of all available findings, with the purpose of considering whether it was global haemostatic function or traditional measures of clotting which was giving the better indication of true disease state. Any results will be tabulated and summarised narratively.

\section{Investigations of heterogeneity}

If there is a sufficient number of studies we investigate heterogeneity. With respect to test accuracy results, we will assume that important heterogeneity beyond that accounted for by chance will be present and will need to be investigated. Our approach will be to perform subgroup analyses using the analytical framework detailed below, using HSROC models.

The provisional framework for investigating heterogeneity will include:

- Type of global measure of haemostatic function (TEG/ ROTEM)

- Time blood sample taken relative to trauma $(\leq 1 \mathrm{~h} />1 \mathrm{~h})$

- Nature of reference standard (INR/PTr of $>1.2$; INR/PTr of $>1.5)$

- Prevalence of TIC (excluding case-control studies if these are included)

- Participant type, especially severity of trauma and mechanism of injury (blunt/penetrating)

- Setting (military or civilian)

- Whether trauma was associated with massive transfusion (yes or mixed/no)

- Case-control study design (if these are included)

- Other aspects of study quality, particularly blinding of the index test and reference standard.

We have no specific plans to investigate heterogeneity of the data concerning uninterpretables or conduct further investigation of discrepant results.

\section{Sensitivity analyses}

In the unlikely event that heterogeneity is not present and the effect of important covariates has not already been analysed, we will investigate the robustness of any summary estimates of test accuracy to the aspects of study quality indicated in the framework for investigating heterogeneity above.

\section{Assessment of reporting bias}

We will not assess reporting bias because its impact in test accuracy is unclear and the tools for investigating it are in the early stages of development.

\section{ACKNOW LEDGEMENTS}

Thanks to Roger Luddington for the use of his diagram of the thromboelastic trace. Thanks also to Jenny Lowe for providing administrative support. 


\section{R E F E R E N C E S}

\section{Additional references}

\section{Barts \& The London 2011}

Barts, The London School of Medicine, Dentistry. The science of the bleeding obvious! Trauma surgery. Summer Science Exhibition 2011, The Royal Society 5-10 July 2011.

\section{Bayliss 2008}

Bayliss SE, Davenport, C. Locating systematic reviews of test accuracy studies: how five specialist review databases measure up. International Journal of Technology Assessment in Health Care 2008;24(4):403-11.

\section{Brohi 2003}

Brohi K, Singh J, Heron M, Coats T. Acute traumatic coagulopathy. The Journal of TRAUMA: Injury, Infection and Critical Care 2003;54(6):1127-30.

de Vet 2008

de Vet HCW, Eisinga A, Riphagen I, Aertgeerts B, Pewsner D. Chapter 7: Searching for studies. In: Deeks JJ, Bossuyt PM, Gatsonis M, Constantine A editor(s). Cochrane Handbook for Systematic Reviews of Diagnostic Test Accuracy. 0.4. The Cochrane Collaboration, updated September 2008.

\section{Doran 2010}

Doran CM, Woolley T, Midwinter MJ. Surgeon General's Operational Policy Letter 10/07 - Management of massive haemorrhage on operations. Defence Medical Services Department 05/01/02, dated June 14, 2007 quoted in Feasibility of Using rotational Thromboelastometry to Assess Coagulation Status of Combat Casualties in a Deployed setting. The Journal of TRAUMA: Injury, Infection and Critical Care 2010;69(1 July Suppl):40-8.

\section{Doust 2005}

Doust JA, Pietrzak E, Sanders S, Glasziou PP. Identifying studies for systematic reviews of diagnostic tests was difficult due to the poor sensitivity and precision of methodologic filters and the lack of information in the abstract. Journal of Clinical Epidemiology 2005;58:444-9.

\section{Dzik 2004}

Dzik WH. Predicting hemorrhage using preoperative coagulation screening assays. Current Hematology Reports 2004;3(5):324-30.

\section{Fraser 2006}

Fraser C, Mowatt G, Siddiqui R, Burr J. Searching for diagnostic test accuracy studies: an application to screening for open angle glaucoma (OAG). Cochrane Colloquium Abstracts Journal. 14th Cochrane Colloquium, Dublin, 2326 October. 2006

Frith 2010

Frith D, Goslings JC, Gaardner C, Maegele M, Cohen MJ, Allard S, et al.Definition and drivers of acute traumatic coagulopathy: clinical and experimental investigations. Journal of Thrombosis and Haemostasis 2010;8:1919-25.

\section{Ganter 2008}

Ganter MT, Hofer CK. Coagulation monitoring: current techniques and clinical use of viscoelastic point of care coagulation devices. International Anaesthesia Research Society 2008;106(5):1366-75.

\section{Greenhalgh 2005}

Greenhalgh T, Peacock R. Effectiveness and efficiency of search methods in systematic reviews of complex evidence: audit of primary sources. BMJ 2005;331(7524):1064-5.

Hess 2009

Hess JR, Lindell AL, Stansbury LG, Dutton RP, Scalea TM. The prevalence of abnormal results of conventional coagulation tests on admission to a trauma center. Transfusion 2009;49(1):34-9.

Holcomb 2007

Holcomb JB, McMullin NR, Pearse L, Caruso J, Wade CE, Oetjen-Gerdes L, et al.Causes of death in U.S. Special Operations forces in the global war on terrorism. Annals of Surgery 2007;245(6):968-91.

Leeflang 2006

Leeflang MMG, Scholten RJPM, Rutjes AWS, Reitsma JB, Bossuyt PMM. Use of methodological search filters to identify diagnostic accuracy studies can lead to the omission of relevant studies. Journal of Clinical Epidemiology 2006;59 (3):234-40.

MacLeod 2003

MacLeod JB, Lynn M, McKenney MG, Dohn SM, Murthda M. Early coagulopathy predicts mortality in trauma. The Journal of TRAUMA: Injury, Infection and Critical Care 2003;55(1):39-44.

Maegele 2007 Maegele M, Lefering R, Yucel N, Tjardes T, Rixen D, Paffrath T, et al.Early coagulopathy in multiple injury: an analysis from the German Trauma Registry on 8724 patients. Injury 2007;38(3):298-304.

Moher 2000

Moher D, Pham B, Klassen TP, Schulz KF, Berlin JA, Jadad $\mathrm{AR}$, et al. What contributions do languages other than English make on the results of meta-analyses?. Journal of Clinical Epidemiology 2000;53(9):964-72.

\section{Park 2009}

Park MS, Martini WZ, Dubick A, Salinas J, Butenas $S$, Kheirabadi B, et al.Thromboelastography as a better indicator of hypercoagulable state after injury than prothrombin time or activate partial thromboplastin time. Journal of Trauma 2009;67(2):266-75.

\section{Reitsma 2009}

Reitsma JB, Rutjes AWS, Whiting P, Vlassov VV, Leeflang MMG, Deeks JJ. Chapter 9: Assessing methodological quality. In: Deeks JJ, Bossuyt PM, Gatsonis C editor(s). Cochrane Handbook for Systematic Reviews of Diagnostic Test Accuracy. 1.0.0. The Cochrane Collaboration, 2009. 
RevMan 2012

The Nordic Cochrane Centre. Review Manager (RevMan).

5.2. Copenhagen: The Cochrane Collaboration, 2012.

\section{Spinella 2009}

Spinella PC, Holcomb JB. Resuscitation and transfusion principles for traumatic hemorrhagic shock. Blood Reviews 2009;23:231-40.

\section{Stainsby 2006}

Stainsby D, MacLennan S, Thomas D, Isaac J, Hamilton PJ. Guidelines on the management of massive blood loss. British Journal of Haematology 2006;135:634-41.

\section{Vincent 2003}

Vincent S, Greenley S, Beaven O. Clinical evidence diagnosis: developing a sensitive search strategy to retrieve diagnostic studies on deep vein thrombosis: a pragmatic approach. Health Information \& Libraries Journal 2003;20 (3):150-9.

\section{Whiting 2011}

Whiting P, Westwood M, Beynon R, Burke M, Sterne JA, Glanville J. Inclusion of methodological filters in searches for diagnostic test accuracy studies misses relevant studies. Journal of Clinical Epidemiology 2011;64(6):602-7.

* Indicates the major publication for the study

\section{A D DITIONAL TABLES}

Table 1. Normal reference values for ROTEM and TEG

\begin{tabular}{|c|c|c|}
\hline & TEG & ROTEM \\
\hline Clotting time (period to $2 \mathrm{~mm}$ amplitude) & $\begin{array}{l}\mathrm{R} \text { (reaction time) } \\
N \text { (whole blood) } 4 \text { to } 8 \mathrm{~min} \\
N \text { (Cit, kaolin) } 3 \text { to } 8 \mathrm{~min}\end{array}$ & $\begin{array}{l}\text { CT (clotting time) } \\
N \text { (Cit, in-TEM) } 137 \text { to } 246 \mathrm{~s} \\
N(\text { Cit, ex-TEM) } 42 \text { to } 74 \mathrm{~s}\end{array}$ \\
\hline $\begin{array}{l}\text { Clot kinetics (period from } 2 \text { to } 20 \mathrm{~mm} \text { am- } \\
\text { plitude) }\end{array}$ & $\begin{array}{l}K \text { (kinetics) } \\
N \text { (WB) } 1 \text { to } 4 \mathrm{~min} \\
N \text { (Cit, kaolin) } 1 \text { to } 3 \mathrm{~min}\end{array}$ & $\begin{array}{l}\text { CFT (clot formation time) } \\
N(\text { Cit, in-TEM) } 40 \text { to } 100 \mathrm{~s} \\
N(\text { Cit, ex-TEM } 46 \text { to } 148 \mathrm{~s}\end{array}$ \\
\hline Clot strengthening (alpha angle) & $\begin{array}{l}\alpha \text { (slope between } \mathrm{r} \text { and } \mathrm{k}) \\
N(\mathrm{WB}) 47^{\circ} \text { to } 74^{\circ} \\
N(\text { Cit, kaolin }) 55^{\circ} \text { to } 78^{\circ}\end{array}$ & $\begin{array}{l}\alpha \text { (slope of tangent at } 2 \mathrm{~mm} \text { amplitude }) \\
N(\text { Cit, in-TEM }) 71^{\circ} \text { to } 82^{\circ} \\
N(\text { Cit, ex-TEM }) 63^{\circ} \text { to } 81^{\circ}\end{array}$ \\
\hline Amplitude (at set time) & $A$ & $A$ \\
\hline Maximum strength & $\begin{array}{l}\text { MA (maximum amplitude) } \\
N \text { (WB) } 55 \text { to } 73 \mathrm{~mm} \\
N \text { (Cit, kaolin) } 51 \text { to } 69 \mathrm{~mm}\end{array}$ & $\begin{array}{l}\text { MCF (maximum clot firmness) } \\
N \text { (Cit, in-TEM) } 52 \text { to } 72 \mathrm{~mm} \\
N \text { (Cit, ex-TEM) } 49 \text { to } 71 \mathrm{~mm} \\
N \text { (Cit, fib-TEM) } 9 \text { to } 25 \mathrm{~mm}\end{array}$ \\
\hline Lysis (at fixed time) & CL30, CL60 & LY30, LY60 \\
\hline
\end{tabular}

TEG: $N$ = normal values for kaolin activated TEG in native whole blood (WB) or citrated and recalcified blood samples (Cit).

ROTEM: $N=$ normal values for contact (partial thromboplastin phospholipids, INTEM), tissue factor (EXTEM) and tissue factor plus platelet inhibitor cytochalasin D (FIBTEM) activated citrated and recalcified blood samples.

Reference values depend on reference population, blood sampling technique, other preanalytical factors, and coagulation activator (Ganter 2008). 
A P P E N DICES

Appendix I. Results interpretation of the thromboelastic trace See Figure 3. 
Figure 3. Viscoelastic haemostatic assays terminology and parameters. Reproduced by kind permission $\circ \alpha$, alpha angle; from Dr Roger Luddington, Addenbrooke's Hospital.

AUC, area under the curve;

CFT, clot formation time;

$\circ$ CL (t), clot lysis (at time t);

- CT, clot time;

$\mathbf{k}$, rate of clot formation;

LY (t), lysis (at time $t$ );

MA, maximum amplitude;

MAXV, maximum velocity;

MAXV-t, time to maximum velocity;

MCF, maximum clot firmness;

MCF-t, time to maximum clot firmness;

ML, maximum lysis;

$r$, time to clot initiation;

ROTEM, Rotational Thromboelastogram;

RT, reaction time;

TEG, Thromboelastograph;

TMA, time to maximum amplitude;

,- no equivalent parameter.

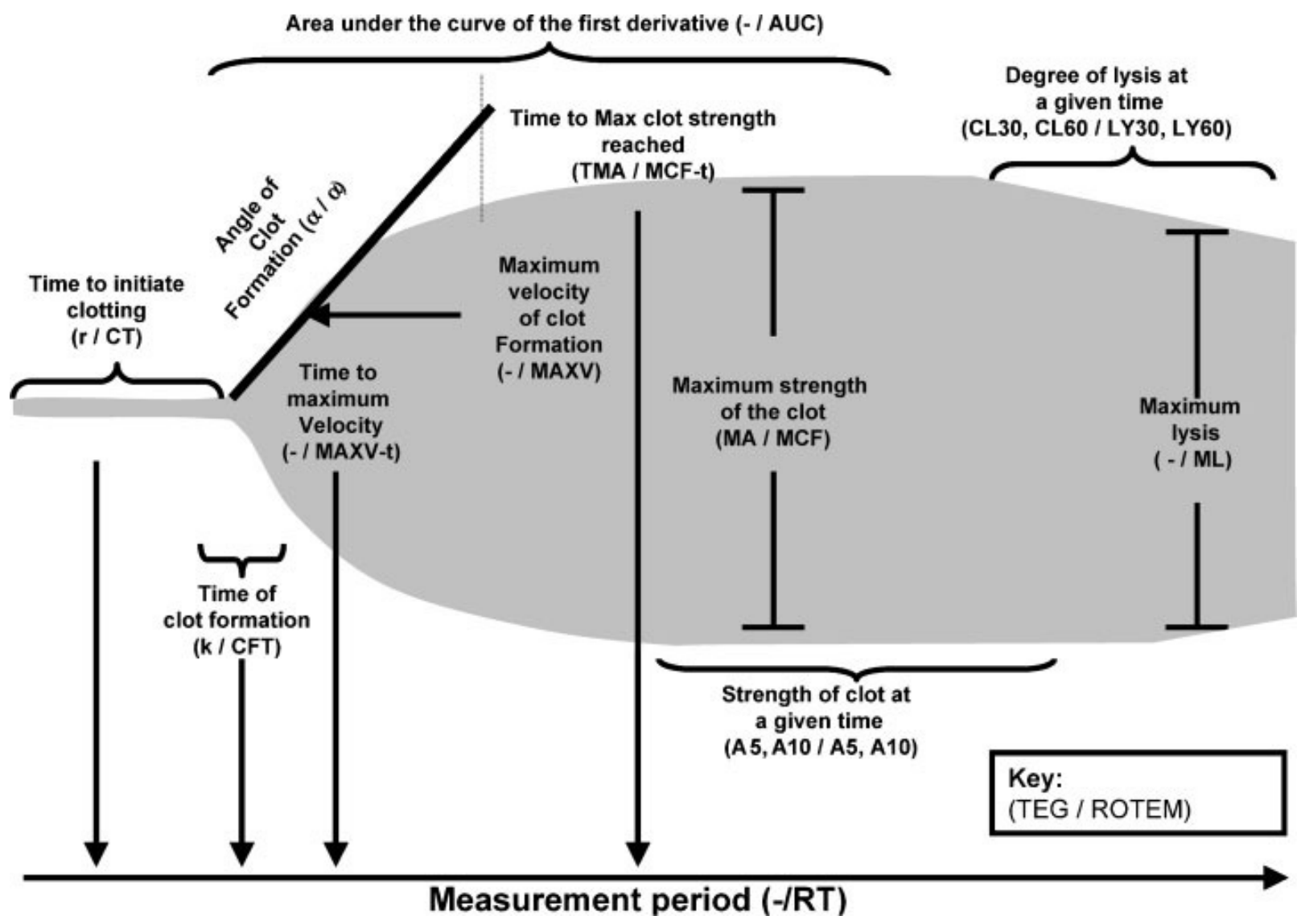

Thromboelastography (TEG) and thromboelastometry (ROTEM) for trauma

induced coagulopathy in adult trauma patients with bleeding (Protocol)

Copyright (? 2013 The Cochrane Collaboration. Published by John Wiley \& Sons, Ltd. 


\section{Appendix 2. TEG and ROTEM equivalent methods}

\section{Clotting time}

In TEG, clotting time is measured as $\mathrm{R}$ (reaction time), $\mathrm{N}$ (whole blood) (normal values for kaolin activated TEG in whole blood) and $\mathrm{N}$ (Cit, kaolin) (normal values for kaolin activated TEG in citrated and recalcified blood). In ROTEM, clotting time is measured as CT (clotting time), N (Cit, INTEM) (normal values for contact) and N (Cit, EXTEM) (normal values for tissue factor).

\section{Clot formation}

Clot formation is measured in TEG as K (kinetics) and - as above - N (whole blood) and N (Cit, kaolin). In ROTEM, CFT (clot formation time) is measured as before as $\mathrm{N}$ (Cit, INTEM) and $\mathrm{N}$ (Cit, EXTEM).

\section{Clot strengthening}

Clot strengthening is measured in both TEG and ROTEM as the alpha angle - in TEG this is defined as the slope between R and $\mathrm{K}$ and in ROTEM this is the slope of tangent at $2 \mathrm{~mm}$ amplitude. Again, both tests give the alpha angle as $\mathrm{N}$ (whole blood) and $\mathrm{N}$ (Cit, kaolin) for TEG and N (Cit, INTEM) and N (Cit, EXTEM) for ROTEM.

\section{Amplitude of clot}

Amplitude of clot (or 'A') is given at set times in both tests.

\section{Maximum clot firmness}

The maximum strength of the clot is measured in TEG as maximum amplitude (MA) and in ROTEM as maximum clot firmness (MCF), and both tests give this measurement both as N (whole blood) and N (Cit, kaolin) for TEG and N (Cit, INTEM) and N (Cit, EXTEM) for ROTEM - although ROTEM also reports tissue factor plus platelet inhibitor cytochalasin D (Cit, FIBTEM).

\section{Clot lysis}

Both tests give readings for clot lysis as CL in TEG (e.g. CL30, CL60) and LY in ROTEM (e.g. LY30, LY60).

\section{Appendix 3. Search strategy}

\section{Search approach}

Despite the work by Vincent 2003, who found high rates of inclusion using a bespoke diagnostic test accuracy (DTA) filter, there remain noted difficulties in searching for and locating DTA studies (Vincent 2003; Doust 2005; Leeflang 2006; Bayliss 2008; de Vet 2008).

With these points in mind, this annexe details our search approaches in this review.

Thromboelastography (TEG) and thromboelastometry (ROTEM) for trauma 


\section{The strategy}

Whiting 2011 found that a search approach which used clusters of 'condition' and 'index test' search terms offered the best sensitivity to a DTA search (Whiting 2011): a view shared by the latest edition of the Cochrane DTA Handbook (de Vet 2008).

In reference to the above, our search strategy uses only the index terms, and will be run without methodological filters, with a view to conducting a highly sensitive search. The search strategy has been peer reviewed by the review authors to ensure that clinical concepts have been addressed.

Database: MEDLINE

Host: Ovid

Date parameters: 1946 to November Week 12012

Date searched: Thursday, November 8th 2012

Searcher: CC

Strategy checked by: $\mathrm{HH}$

Strategy:

\section{\# Searches}

Results

1 (Thrombelastogra $\$$ or Thromboelastogra $\$$ or (thromb $\$$ adj2 elastogra $\$$ ) or TEG or haemoscope or haemonetics).mp

2 Thrombelastography/

3 (thromboelastom $\$$ or thrombelastom $\$$ or (thromb $\$$ adj2 elas- 338 tom\$) or (rotational adj2 thrombelast) or ROTEM or "tem international").mp

41 or 2 or 3

5 limit 4 to $y r=$ " 1970 -Current" 3602

6 exp Animals/ not Humans.sh. 3807580

$75 \operatorname{not} 6$

Hits: 3124

Notes: N/A

File Name: Medline3124.txt

\section{Search limits}

As suggested by the Cochrane Handbook, and by others, the search will not be limited by language, in order to avoid a potential language bias (Moher 2000; de Vet 2008). The search will be limited to 'human only' populations and by date, '1970 to current'.

\section{Search resources}

We have chosen the following resources to search for this review as they reflect the most likely places to find DTA studies. This list has been cross-checked against other DTA reviews, and includes literature searching to identify any additional resources which might be used. Bayliss 2008 and Fraser 2006 were of particular use here.

\section{Database resources}

- British Nursing Index (BNI) via ProQuest

Thromboelastography (TEG) and thromboelastometry (ROTEM) for trauma

induced coagulopathy in adult trauma patients with bleeding (Protocol)

Copyright (? 2013 The Cochrane Collaboration. Published by John Wiley \& Sons, Ltd. 
- Centre for Reviews and Dissemination (CRD) through http://www.crd.york.ac.uk/crdweb/SearchPage.asp

- CINAHL via EBSCO

- The Cochrane Library through http://www.thecochranelibrary.com

- Conference Proceedings Citation Index- Science (CPCI-S) via ISI*

- Conference Proceedings Citation Index- Social Science \& Humanities (CPCI-SSH) via ISI*

- EMBASE via OVID

- HMIC via OVID

- MEDLINE in Process via OVID

- MEDLINE via OVID

- PsycINFO via OVID

- Science Citation Index Expanded (SCI-EXPANDED) via ISI*

- Social Sciences Citation Index (SSCI) via ISI*

- Transfusion Evidence Library via www.transfusionevidencelibrary.com

- Current Controlled Trials via http://www.controlled-trials.com/

* these resources will be searched through the Web of Science interface hosted by ISI.

\section{Web resources}

Bayliss 2008 reviewed the following internet resources for locating DTA systematic reviews. This review also included DARE and HTA, which will be searched as part of the approach detailed above (Bayliss 2008).

We will include an internet search on Google, the manufacturer websites for the index tests relevant to this review, and the Cochrane DTA group website. Given the nature of our search, and the specific nature of what we are looking for, generic websites would not seem to be the best tools in locating diagnostic reviews.

- Aggressive Research Intelligence Facility (ARIF) via http://tinyurl.com/3u9tevp

- C-EBLM

- Diagnostic Test Accuracy Working Group (Cochrane) via http://srdta.cochrane.org/

- Google

- MEDION database via http://www.mediondatabase.nl/

- Haemonetics Corporation http://www.haemonetics.com/en.aspx

- TEM Innovations GmbH http://www.rotem.de/site/index.php

\section{Additional searches}

\section{Snowballing}

We will use a combined approach of database searching and supplementary techniques.

Doust 2005 highlights the importance of snowballing in DTA reviews to maximize sensitivity, and the approach identified by Greenhalgh 2005 improved identification of includable studies (Doust 2005; Greenhalgh 2005). Snowballing will be especially important to this review in view of findings by Whiting 2011 that, despite not using a DTA filter, a condition and index search in MEDLINE also missed potential studies (Whiting 2011).

Accordingly, we will employ 'citation chasing' for all included studies identified by our searching. Science Citation Index/Social Science Citation Index will be used for forward citation chasing and the bibliography of each included paper will be screened for retrieval by $\mathrm{HH}$ and $\mathrm{CH}$.

We will attempt to contact authors of included studies.

\section{Updating searches}

We will activate citation alerts on included studies to keep abreast of any developments whilst the review is in process.

We will take a view on running update searches, whilst de-duplicating any citation alerts that have been identified, if/when the technology is updated. The review syntax will be checked for any changes to controlled syntax at this time. These searches will run from the date of the main search and use the same resources as above.

Thromboelastography (TEG) and thromboelastometry (ROTEM) for trauma

- induced coagulopathy in adult trauma patients with bleeding (Protocol)

Copyright (? 2013 The Cochrane Collaboration. Published by John Wiley \& Sons, Ltd. 


\section{Notes}

Any includable, diagnostic test accuracy studies located will be forwarded to the Cochrane Diagnostic Test Accuracy Methods Group Specialised Register.

\section{Appendix 4. QUADAS-2 quality assessment}

(from http://www.bris.ac.uk/quadas/quadas-2 18/05/2012)

See Figure 4, Figure 5 and Figure 6.

Figure 4. QUADAS-2 page I

\section{QUADAS-2}

Phase 1: State the review question:

\begin{tabular}{l} 
Patients (setting, intended use of index test, presentation, prior testing): \\
Adult patients presenting at civilian or military emergency rooms with blunt or penetrating trauma. The index test is intended to identify \\
the presence of coagulopathy. \\
\hline $\begin{array}{l}\text { Index test(s): } \\
\text { Thromboelastography (TEG) and/or thromboelastometry (ROTEM) }\end{array}$ \\
$\begin{array}{l}\text { Reference standard and target condition: } \\
\text { Prothrombin Time ratio (PTr) and/or International Normalised Ratio (INR) - target condition is Trauma-Induced Coagulopathy }\end{array}$
\end{tabular}

Phase 2: Draw a flow diagram for the primary study

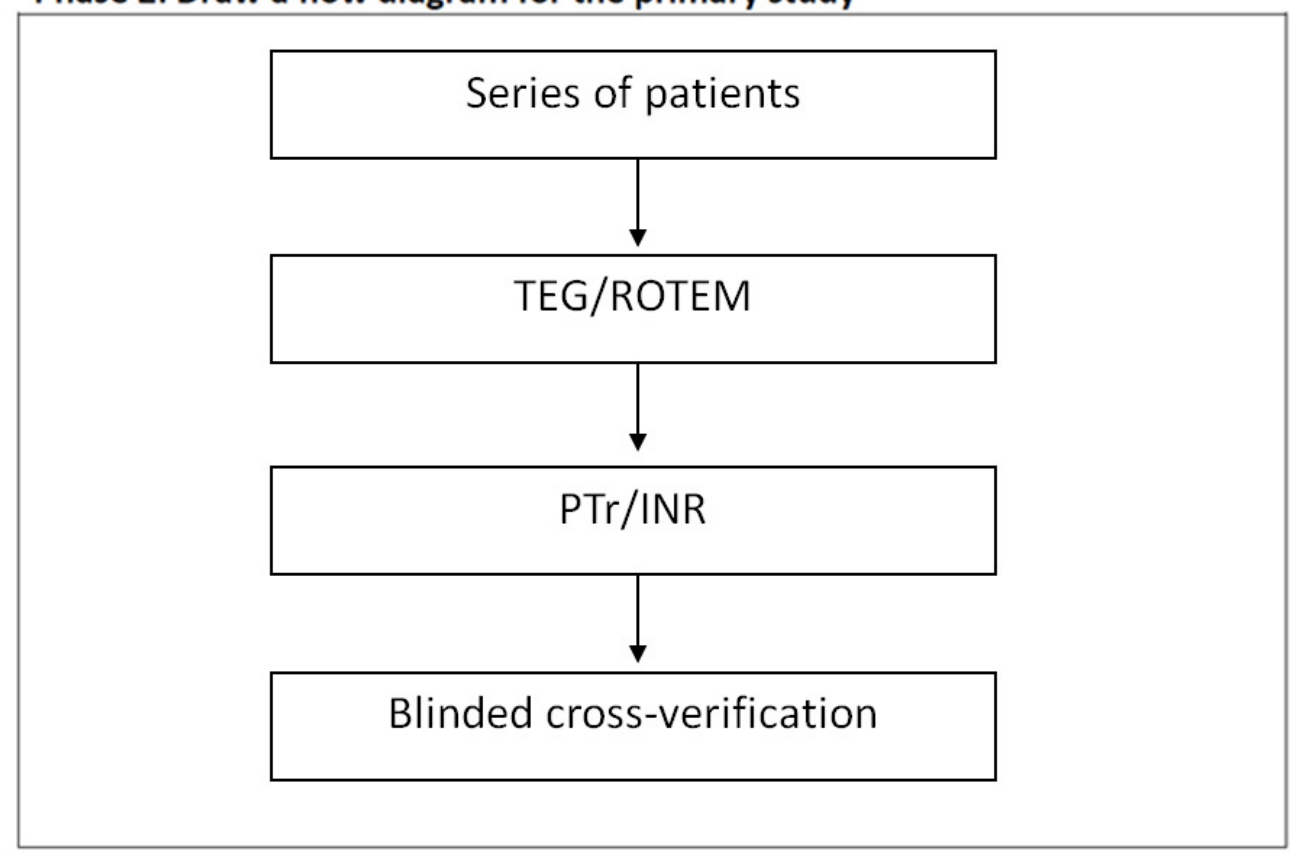

Thromboelastography (TEG) and thromboelastometry (ROTEM) for trauma 
Figure 5. QUADAS-2 page 2

Phase 3: Risk of bias and applicability judgments

QUADAS-2 is structured so that 4 key domains are each rated in terms of the risk of bias and the concern regarding applicability to the research question (as defined above). Each key domain has a set of signalling questions to help reach the judgments regarding bias and applicability.

\section{DOMAIN 1: PATIENT SELECTION}

\section{A. Risk of Bias}

Describe methods of patient selection:

Patients identified by clinicians on admission to the emergency room or field hospital. Random sampling may not be possible in many/any cases due to the environment and treatment needs. Inappropriate exclusions are not considered to be a major risk in these scenarios.

* Was a consecutive or random sample of patients enrolled? $\quad$ Yes/No/Unclear

* Was a case-control design avoided?

$*$ Did the study avoid inappropriate exclusions?

Could the selection of patients have introduced bias?

RISK: LOW/HIGH/UNCLEAR

\section{B. Concerns regarding applicability}

Describe included patients (prior testing, presentation, intended use of index test and setting): Adult trauma patients admitted into the emergency room (military or civilian setting) presenting with blunt or penetrating trauma and clinically-suspected Trauma-Induced Coagulopathy (TIC). Fluids or crystalloids may have been administered at initial attendance, but haemostatic testing will take place on admission and it is these initial samples which would be analysed using the index test.

Is there concern that the included patients do not match CONCERN: LOW/HIGH/UNCLEAR the review question?

\section{DOMAIN 2: INDEX TEST(S)}

If more than one index test was used, please complete for each test.

\section{A. Risk of Bias}

Describe the index test and how it was conducted and interpreted:

TEG - whole-blood sample taken as soon as possible after presentation and analysis carried out point of care by trained nursing staff. the TEG analyser produces a global haemostatic assay in diagrammatic form which is analysed by clinical staff and who treat accordingly.

ROTEM - as above; the difference is in the mechanism (in TEG a pin rotates within a static cup; in ROTEM the pin is static and the cup rotates)

* Were the index test results interpreted without $\quad$ Yes/No/Unclear knowledge of the results of the reference standard?

$*$ If a threshold was used, was it pre-specified?

Could the conduct or interpretation of the index test RISK: LOW /HIGH/UNCLEAR

have introduced bias?

\section{B. Concerns regarding applicability}

Is there concern that the index test, its conduct, or

CONCERN: LOW /HIGH/UNCLEAR

interpretation differ from the review question? 
Figure 6. QUADAS-2 page 3.

DOMAIN 3: REFERENCE STANDARD

A. Risk of Bias

Describe the reference standard and how it was conducted and interpreted:

The reference range we will be using is based on pro-thrombin time ratio (PTr)/International Normalized Ratio (INR), with the lower limit of the

or greater and the upper limit of 1.5 or greater (with the range used depending on individual trauma centre protocols).

This will be conducted as a laboratory-based test by specialist laboratory staff using conventional and established laboratory methods.

$*$ Is the reference standard likely to correctly classify the target $\quad$ Yes/No/Unclear

condition?

* Were the reference standard results interpreted without $\quad$ Yes/No/Unclear

knowledge of the results of the index test?

Could the reference standard, its conduct, or its $\quad$ RISK: LOW /HIGH/UNCLEAR

interpretation have introduced bias?

B. Concerns regarding applicability

Is there concern that the target condition as defined by

the reference standard does not match the review

question?

CONCERN: LOW /HIGH/UNCLEAR

\section{DOMAIN 4: FLOW AND TIMING}

\section{A. Risk of Bias}

Describe any patients who did not receive the index test(s) and/or reference standard or who were excluded from the $2 \times 2$ table (refer to flow diagram):

In the trauma situation there is the chance that in the course of emergency treatment patients do not receive the index tests and/or the reference standard. This may be due to mortality, further complication in the treatment pathway or disruption to the clinical process (such as in active

military settings).

Describe the time interval and any interventions between index test(s) and reference standard:

This cannot be prestated beyond specifying "within 24 hours" - tests take place as soon as the casualty is admitted into hospital, with blood tests taken as soon as possible on presentation.

$*$ Was there an appropriate interval between index test(s)

Yes/No/Unclear

and reference standard?

* Did all patients receive a reference standard?

* Did patients receive the same reference standard?

$*$ Were all patients included in the analysis?

Could the patient flow have introduced bias?

Yes/No/Unclear

Yes/No/Unclear

Yes/No/Unclear

RISK: LOW /HIGH/UNCLEAR

Thromboelastography (TEG) and thromboelastometry (ROTEM) for trauma 
Operationalising QUADAS-2:

When will the index test lead to 'concerns regarding applicability'?

When there is concern that it is being misapplied, used in a situation different from that stated in the research question, or interpreted in a way that is out of the bounds of the research question.

How will we define whether the reference standard is likely to correctly classify the target condition' or not?

If there is evidence of misapplication, e.g. being used in the wrong environment, or generating noticeably different results than those expected without reasonable explanation.

What is the 'appropriate interval' between index and reference tests?

This would be a judgement based upon the professional opinion of our clinical experts.

\section{CONTRIBUTIONSOFAUTHORS}

Writing the first draft of the protocol - Harriet Hunt

Methodological advice - Chris Hyde, Pablo Perel

Content advice - all authors

Editing protocol - all authors

\section{DECLARATIONSOF INTEREST}

None known.

\section{SOURCES OF SUPPORT}

\section{Internal sources}

- Dutch Cochrane Centre, Netherlands.

Training

- Cochrane Diagnostic Test Accuracy Working Group, UK.

Technical support

\section{External sources}

- NHS Blood and Transplant through an NIHR Programme Grant for Applied Research, UK.

Project funding

- NIHR CLAHRC, UK.

We acknowledge support from the National Institute for Health Research (NIHR) Collaboration for Leadership in Applied Health Research and Care (CLAHRC) for the South West Peninsula. The views expressed in this publication are those of the author(s) and not necessarily those of the NHS, the NIHR or the Department of Health in England.

Thromboelastography (TEG) and thromboelastometry (ROTEM) for trauma 